\author{
Tansel Terzi \\ Başak Karakurum \\ Serap Üçler \\ Levent E. Inan \\ Cankat Tulunay
}

\section{Greater occipital nerve blockade in migraine, tension-type headache and cervicogenic headache}

Received: 31 January 2002

Accepted in revised form: 2 July 2002

T. Terzi • B. Karakurum (凶) • S. Üçler

L.E. Inan

Department of Neurology,

Ministry of Health Ankara Research and

Training Hospital,

Ankara, Turkey

Tel.: +90-322-454-7613

Fax: +90-322-327-1273

e-mail: bkarakurum@hotmail.com

C. Tulunay

Department of Pharmacology,

Medical Faculty,

Ankara University, Ankara, Turkey

\begin{abstract}
The blockade of the greater occipital nerve (GON) has been used in the treatment of migraine without aura (MWOA), tension-type headache (TTH) and cervicogenic headache $(\mathrm{CH})$. There have been only a few reports about the effectiveness of the GON blockade in patients with MWOA and TTH and it has not yet been clarified whether or not it is a diagnostic tool for $\mathrm{CH}$. In this study, we therefore investigated the diagnostic value of GON blockade in patients with $\mathrm{CH}, \mathrm{MWOA}$ and TTH. Sixty patients who were affected by TTH, MWOA and $\mathrm{CH}$ participated in the study. They were divided into three main groups, each of which consisted of 20 patients with TTH, MWOA and
\end{abstract}

$\mathrm{CH}$ respectively. Each group was then divided into two sub-groups with 10 patients, ten of whom were injected with $1 \mathrm{ml} 2 \%$ prilocaine, and the other ten with $1 \mathrm{ml}$ physiological saline (PS). Our results showed that GON blockade reduced pain in the orbitofrontal (OF) and orbitonuchal (ON) areas in patients with $\mathrm{CH}$. In MWOA and TTH patients, GON blockade reduced pain only in the $\mathrm{ON}$ area. In the light of these findings, we may conclude that GON blockade is a diagnostic tool if it is effective in the $\mathrm{ON}$ and $\mathrm{OF}$ areas.

Key words Greater occipital nerve blockade $\cdot$ Migraine $\cdot$ Tension-type headache Cervicogenic headache

\section{Introduction}

The diagnostic value of greater occipital nerve (GON) blockade in patients with cervicogenic headache $(\mathrm{CH})$ is well known $[1,2]$. However, there are few reports about the usefulness of GON blockade in patients with migraine without aura (MWOA) and tension-type headache (TTH) [3-5].

The greater occipital nerve contains fibres from the dorsal ramus of the $\mathrm{C} 2$ root and $\mathrm{C} 3$ occipital nerve. Afferent stimulation from joints or $\mathrm{C} 1-\mathrm{C} 3$ roots may activate the trigeminal sensory fibres in the brainstem. Hence, the trigeminal vascular system plays a central role in the genesis of headache. This can be a possible explanation for the effectiveness of GON blockade in different headache types $[1,2]$.

Cervicogenic headache is a unilateral headache, which initially may be remitting but tends to become chronic over time. Pain, stemming from the neck, usually spreads to the oculofrontotemporal area [3, 4]. It is moderate in intensity, appears more frequently in women, and is associated with signs and symptoms linking it to the neck $[4,5]$. Although unilaterality of pain was a strict criterion as defined in the original criteria for $\mathrm{CH}$ diagnosis, recent reports proposed that it might also be bilateral [3]. Not only GON but also C2/C3 blockades are effective in patients with $\mathrm{CH}$ [6].

There are still some unresolved questions about the differential diagnosis of $\mathrm{CH}$, TTH and MWOA. Although some reports indicated that anaesthetic blockade of GON is accepted as a diagnostic tool for $\mathrm{CH}$, some studies showed the effectiveness in patients with TTH or MWOA [4, 7-10]. The aim of this placebo-controlled, randomised and double-blind study was to evaluate the diagnostic value of GON blockade in patients with $\mathrm{CH}$, TTH and MWOA. 


\section{Materials and methods}

A total of 60 consecutive headache patients were enrolled. Inclusion criteria were:

1. The presence of a primary headache disorder (migraine or TTH) or cervicogenic headache. MWOA and TTH were diagnosed according to the International Headache Society (IHS) headache classification [11] and $\mathrm{CH}$ was diagnosed according to the 1998 criteria of Sjaastad et al. [7].

2. The specificity of unilateral GON irritation and therefore the strict unilaterality of pain. In this regard TTH generally has bilateral character, even though it may be unilateral in $5 \%-26 \%$ of the cases $[12,13]$.

3. Pain referred when the GON area $(2 \mathrm{~cm}$ laterally and $2 \mathrm{~cm}$ below the protuberantia occipitalis externa) was pressed. We only included patients with a predominantly unilateral headache [13]. According to IHS criteria, 20 patients could be classified as suffering from MWOA and 20 from TTH. The remaining 20 patients were affected by $\mathrm{CH}$ according to 1998 Sjaastad et al.'s criteria [7]. All patients had a unilateral location of head pain and referred pain in the GON area.

The study used a double-blind design and involved two neurologists. Ten patients with each headache type were randomly assigned to receive a $1-\mathrm{ml}$ injection of $2 \%$ prilocaine in physiological $(0.9 \%)$ saline (treatment group) or a 1-ml injection of saline (placebo control group). One of the neurologists prepared the prilocaine and placebo solutions, while the patients were examined and injected by another neurologist who was blind to the treatments as were the patients. The patients gave their informed consent to the design and technique of the study.

We wanted to determine the specificity of unilateral GON irritation in the three groups. Therefore, unilaterality and GON irritation were the criteria for admission to this study.

All patients were asked to keep a headache diary in which they reported the number of days with headache, localisation, and intensity of head pain, the occurrence of accompanying symptoms (e.g. nausea, vomiting, photophobia and phonophobia), and whether the pain worsened with routine daily activity.

The GON blockade technique was applied according to Saadah and Taylor [14]. The patient was asked to lie face downwards on the table. The hair above and below the superior nuchal line was separated using 1-inch thick paper tape. The scalp was cleaned with iodine and a 22-gauge needle was used. It was inserted to the occipital bone, slightly withdrawn, and then prilocaine or the placebo solution was injected. The patient had to lie down for half an hour after the injection to avoid the dizzines, that usually occurs when the occipital muscles are anaesthetized. Headache severity in the orbitofrontal (OF) and orbitonuchal $(\mathrm{ON})$ areas was assessed on a visual analogue scale (VAS) from 0 (no pain) to 10 (intense pain) before and 5, 10 and 30 minutes after GON blockade. If the VAS decreased $50 \%$ or more, we considered GON blockade to be effective.

Mann-Whitney U test, Wilcoxon test and $t$ test were used for statistical analysis. A $p$ value $<0.01$ was accepted to be statistically significant.

\section{Results}

The characteristics of the 60 patients are reported in Table 1. For all headache types, no statistically significant difference existed in headache duration or frequency between the placebo and prilocaine groups

Data concerning the VAS score referred to the ON and OF areas are reported in Tables 2 and 3, respectively. In the $\mathrm{CH}$ group, there was no significant difference in pain intensity between prilocaine and placebo groups for either the $\mathrm{OF}$ or the $\mathrm{ON}$ area at time $0(\mathrm{U}=32, p>0.01$ and $\mathrm{U}=35.5, p>0.01$, respectively). A significant decrease in pain was found after local anaesthetic (LA) injection in both the $\mathrm{OF}$ and $\mathrm{ON}$ areas at 5, 10 and 30 minutes compared to placebo $(p<0.01)$.

We observed that the $\mathrm{CH}$ group with local anaesthetic was significantly improved in the $\mathrm{OF}$ and $\mathrm{ON}$ areas. Thirty minutes after GON blockade, the severity of pain decreased in the OF area $(72.59 \%)$ and in the ON area $(77.72 \%)$. In the placebo group, the decrease of pain severity in the OF and $\mathrm{ON}$ areas was $8.01 \%$ and $9.26 \%$, respectively. We found that there was no difference between local anaesthetic and placebo in the OF area in the MWOA patient group. Although the reduction in the severity of pain at 30 minutes was $14.7 \%$ in the OF and $40.85 \%$ in the ON areas, in the local anaesthetic group there was no statistically significant decrease when compared to the placebo group. We observed the reduction of pain severity in the ON area of the TTH group (54.82\%)

Table 1 Characteristic of 60 patients by headache type and treatment group. Each group had 10 subjects

\begin{tabular}{|c|c|c|c|c|c|c|}
\hline & \multicolumn{2}{|c|}{ Cervicogenic } & \multicolumn{2}{|c|}{ Migraine } & \multicolumn{2}{|c|}{ Tension-type } \\
\hline & Prilocaine & Placebo & Prilocaine & Placebo & Prilocaine & Placebo \\
\hline Age, years & $42.0(7.9)$ & $39.7(7.7)$ & $33.7(2.9)$ & $33.9(6.1)$ & $29.8(6.3)$ & $31.7(5.7)$ \\
\hline Men, n (\%) & $1(10)$ & $1(10)$ & $0(0)$ & $0(0)$ & $9(90)$ & $10(100)$ \\
\hline Headache frequency, $\mathrm{n} /$ month & $9.7(8.5)$ & $9.6(4.0)$ & $4.2(2.1)$ & $3.9(1.6)$ & $15.4(7.8)$ & $15.1(5.6)$ \\
\hline Headache duration, hours & $21.3(9.9)$ & $18.2(7.2)$ & $8.7(3.0)$ & $7.6(2.3)$ & $12.7(3.8)$ & $13.1(2.8)$ \\
\hline
\end{tabular}


Table 2 Headache severity in the orbitofrontal area during blockade of the greater occipital nerve (GON) as assesed on a visual analogue scale (VAS) from 0 (no pain) to 10 (intense pain), by treatment group. Values are mean (standard deviation)

\begin{tabular}{lll}
\hline & Prilocaine & Placebo \\
\hline Cervicogenic headache & & \\
$\quad$ Baseline & $6.60(0.84,0.27)$ & $7.20(0.92,0.29)$ \\
5 min & $4.40(1.17,0.97)$ & $6.60(0.97,0.31)$ \\
10 min & $3.10(0.99,0.31)$ & $6.50(1.08,0.34)$ \\
30 min & $1.70(1.57,0.50)$ & $6.60(0.97,0.31)$ \\
Migraine without aura & & $9.20(0.79,0.25)$ \\
Baseline & $8.20(0.79,0.25)$ & $8.70(1.34,0.42)$ \\
5 min & $8.00(0.94,0.30)$ & $8.70(1.16,0.37)$ \\
10 min & $7.70(1.25,0.40)$ & $8.80(1.14,0.36)$ \\
30 min & $7.10(2.47,0.78)$ & $5.70(0.67,0.21)$ \\
Tension-type headache & & $5.60(0.70,0.22)$ \\
Baseline & $5.80(0.42,0.13)$ & $5.30(0.95,0.30)$ \\
5 min & $5.00(0.82,0.26)$ & $5.20(1.03,0.33)$ \\
10 min & $4.40(1.26,0.40)$ & \\
\hline
\end{tabular}

Table 3 Headache severity in the orbitonuchal area during blockade of the greater occipital nerve (GON), as assesed on a visual analogue scale from 0 (no pan) to 10 (intense pain), by treatment group. Values are mean (standard deviation)

\begin{tabular}{lll}
\hline & Prilocaine & Placebo \\
\hline $\begin{array}{l}\text { Cervicogenic headache } \\
\text { Baseline }\end{array}$ & $6.70(0.97,0.30)$ & $7.20(0.95,0.29)$ \\
5 min & $4.00(0.95,0.47)$ & $6.40(0.93,0.27)$ \\
10 min & $2.40(1.49,0.37)$ & $6.40(0.84,0.27)$ \\
30 min & $1.40(1.17,0.50)$ & $6.40(0.84,0.27)$ \\
Migraine without aura & & $8.70(0.67,0.21)$ \\
Baseline & $8.00(1.05,0.33)$ & $7.60(1.26,0.40)$ \\
5 min & $6.00(2.54,0.80)$ & $6.90(1.66,0.53)$ \\
10 min & $5.40(2.91,0.92)$ & $6.80(2.20,0.70)$ \\
30 min & $4.80(3.12,0.99)$ & \\
Tension-type headache & & $6.10(0.88,0.28)$ \\
Baseline & $6.00(0.94,0.30)$ & $5.50(0.85,0.27)$ \\
5 min & $4.40(1.84,0.58)$ & $5.10(0.99,0.31)$ \\
10 min & $3.30(2.06,0.65)$ & $4.80(1.48,0.47)$ \\
30 min & $2.80(2.35,0.74)$ & \\
\hline
\end{tabular}

at 30 minutes. There was no difference between local anaesthetic and placebo in the OF area.

The severity of pain in the OF area decreased more significantly in the $\mathrm{CH}$ group than in the TTH group ( $p<0.01$, Mann Whitney U-Wilcoxon test), whereas the decrease of pain severity in the $\mathrm{ON}$ area was similar in both headache groups $(p<0.01$ both groups, Mann Whitney U-Wilcoxon test). The decrease in the severity of pain in the OF and ON areas was also more significant in $\mathrm{CH}$ than in MWOA.

\section{Discussion}

The GON contains fibres from $\mathrm{C} 2$ and $\mathrm{C} 3$. The fibres emerge through the trapezius and sternocleidomastoid muscles, and infratentorial intracranial structures are innervated by the C1-C3 nerves [6-8]. The C2-C3 dorsal roots, which supply the occipital nerves with the sensory fibres, also communicate in the cervical cord with the cau- 
dal parts of the trigeminal nerve nucleus. The trigeminal vascular system plays a central role in the genesis of headache. When trigeminal pathways are stimulated, extracranial vessels dilate and cause nasal congestion and flushing. Stimulation of the trigeminal system causes local release of substance $\mathrm{P}$, histamine, serotonin and prostaglandins, and innervates autonomic pathways in the cranial vasculature. Hence, this is a possible explanation of how GON causes headache [1,7-10].

In the light of these theories, GON blockade has been used in the treatment of different headache types. GON irritation has been observed in $30 \%$ of patients with idiopathic headache [2]. Additionally, blockade of the painproducing nerve with an anaesthetic substance has been used for a long time $[13,14]$. Although the effectiveness and diagnostic value of GON blockade in $\mathrm{CH}$ patients has been well known, it has not been clarified whether or not it is a diagnostic tool for $\mathrm{CH}$, since there have been few reports in this regard. Only a few reports exist about the value and effectiveness in patients with MWOA and TTH [10, 15-17]. Bovim and Sand studied the therapeutic blockade of GON and supraorbital nerve blockade in $\mathrm{CH}$, MWOA and TTH [10]. They claimed that the severity of headache decreased in patients with $\mathrm{CH}(54.5 \%)$, TTH (14\%) and MWOA (6\%) [10].

In the light of these studies, we know that if there is tenderness and irritation in the GON area it may be relieved by its blockade. On the other hand, the effectiveness of GON blockade is still obscure since previous reports were not placebo-controlled, double-blind and randomised. In this study, we investigated the value and diagnostic importance of GON blockade in the $\mathrm{CH}$, MWOA and TTH groups. However, some authors have used GON blockade during headache and between attacks. Thus, we believe that this study will contribute to the clarification of this problem.

We observed that the $\mathrm{CH}$ group with local anaesthetic was significantly improved in the $\mathrm{OF}$ and $\mathrm{ON}$ areas.
Relatedly, Bovim and Sand found a $77 \%$ pain reduction after GON blockade in $\mathrm{CH}$ patients [10]; thus, there seems to be a close correlation.

Although the reduction in the severity of pain at 30 minutes was $14.7 \%$ in the $\mathrm{OF}$ and $40.85 \%$ in the ON areas, in the local anaesthetic group there was no statistically significant decrease when compared to the placebo group. These findings show that the pain reduction in the MWOA group in the OF and $\mathrm{ON}$ areas was found to be lower than that in the $\mathrm{CH}$ group with local anaesthetic injection.

We observed the reduction of pain severity in the $\mathrm{ON}$ area of the TTH group (54.82\%) at 30 minutes. These findings were similar but higher than the findings of Bovim and Sand [10].

In this study, we observed that the blockade of GON was more effective in the $\mathrm{CH}$ group than in the other headache patient groups. Furthermore, the reduction of pain severity was observed not only in the $\mathrm{ON}$ area but also in the $\mathrm{OF}$ area. This may be explained by the Kerr principle. Kerr showed that the upper cervical root ganglia synapsed with trigeminal spinal ganglia. Hence, pain from the cervical area is referred to the trigeminal area as OF $[6,7]$.

Using a placebo-controlled, randomised and doubleblind technique, $\mathrm{CH}$, TTH and MWOA responded differently to local anaesthetic and placebo. These results support the hypothesis that different pathogenetic factors may have played a significant role in the three headache groups. Our results show that $\mathrm{CH}$, MWOA and TTH responded differently to GON blockade during headache attack. The blockade was more effective in $\mathrm{CH}$ patients than in MWOA and TTH patients.

In conclusion, the blockade of GON is effective and useful for the diagnosis of $\mathrm{CH}$. It markedly reduced pain intensity simultaneously in the OF and ON areas. In the MWOA and TTH groups the severity of pain was reduced in the ON area. So, we suggest that GON blockade is a diagnostic tool effective in the OF area.

\section{References}

1. Gawel MJ, Rothbart PJ (1992)

Occipital nerve block in the management of headache and cervical pain. Cephalalgia 12:9-13

2. Bovim G, Fredriksen TA, Stolt-Nielsen A, Sjaastad O (1992) Neurolysis of the greater occipital nerve in cervicogenic headache. A follow up study. Headache 32:175-179

3. Antonaci F, Fredriksen TA, Sjaastad O (2001) Cervicogenic headache: clinical presentation, diagnostic criteria, and differential diagnosis. Curr Pain Headache Rep 5(4):387-392
4. Antonaci F, Ghirmai S, Bono G, Sandrini G, Nappi G (2001) Cervicogenic headache: evaluation of the original diagnostic criteria Cephalalgia 21:573-583

5. Anthony M (2000) Cervicogenic headache: prevalence and response to local steroid therapy. Clin Exp Rheumatol 18[2 Suppl 19]:S59-S64
6. Inan $\mathrm{N}$, Ceyhan $\mathrm{A}$, Inan $\mathrm{L}$, Kavaklioğlu O, Alptekin A, Unal N (2001) C2/C3 nerve blocks and greater occipital nerve block in cervicogenic headache treatment. Funct Neurol 16(3):239-243

7. Sjaastad O, Fredriksen TA, Pfaffenrath V (1998) Cervicogenic headache: diagnostic criteria. The Cervicogenic Headache International Study Group. Headache 38(6):442-445

8. Pearce JM (1995) Cervicogenic headache: a personal view. Cephalalgia 15:463-469 
9. Caputi CA, Firetto V (1997)

Therapeutic blockade of greater occipital and supraorbital nerves in migraine patients. Headache 37:174-179

10. Bovim G, Sand T (1992) Cervicogenic headache, migraine without aura and tension-type headache. Diagnostic blockade of greater occipital and supra-orbital nerves. Pain 51:43-48

11. - (1988) Classification and diagnostic criteria for headache disorders, cranial neuralgias and facial pain. Headache Classificatin Committee of the International Headache Society. Cephalalgia 8[Suppl 7]:1-96
12. Jensen R, Paiva T (1993) Episodic tension type headache In: Olesen J, TfeltHansen P, Welch KMA (eds) The headaches. Raven, New York, pp 497-503

13. Piovesan EJ, Werneck LC, Kowacs PA, Tatsui CE, Lange MC, Vincent M (2001) Anesthetic blockade of the greater occipital nerve in migraine prophylaxis. Arq Neuropsiquiatr 59:545-551

14. Saadah HA, Taylor FB (1987) Sustained headache syndrome associated with tender occipital nerve zones. Headache 27(4):201-205
15. D'Amico D, Leone M, Bussone G (1994) Side-locked unilaterality and pain localization in long-lasting headaches: migraine, tension-type headache, and cervicogenic headache. Headache 34:526-530

16. Bovim G, Berg R, Dale LG (1992) Cervicogenic headache: anesthetic blockades of cervical nerves (C2-C5) and facet joint $(\mathrm{C} 2 / \mathrm{C} 3)$. Pain 49(3):315-320

17. Anthony M (1992) Headache and the greater occipital nerve. Clin Neurol Neurosurg 94:297-301 